\title{
Dynamic state based on autoregressive model for outgoing line of high voltage stations
}

\author{
Yanhong Wang1, a * Tao Xin2,b \\ Hebei normaluniversity,Shijiazhuang,China \\ Hebei normaluniversity,Shijiazhuang,China \\ aemail:wangjinhuisiling@163.com
}

\begin{abstract}
Keywords: autoregressive model. Voltage autoregressive models. power system states.
Abstract. Authors of papers to present a dynamic state prediction method based on an autoregressive model for outgoing Line of high voltage stations of power plants. When load is increased line autoregressive model at a constant power factor, it is proved in this paper that the bus voltages autoregressive models quadratic and the autoregressive model models for predicting the next voltage is based on three prior estimates. This logic is tested on the IEEE-50 bus system. The test results demonstrate that under morning load pick-up, , line opening, the proposed method is correct and gives valid predictions. Theoretical analysis and results show that the proposed method based on autoregressive models has great potential in predicting power system states.
\end{abstract}

\section{Introduction}

The autoregressive models, power system state prediction has been used to improve power system performance and reliability, so it has become important to estimate and predict the state of the power system in order to protect against blackouts. The state of the power system is the collection of all the complex bus voltages. Static state estimation [1] in Energy Management Systems cannot be used to predict transient events. Under such circumstances, Dynamic state estimation using extended filters [2] can be used for system state model identification, state forecasting and state filtering under transient conditions.

Due to the high demand on precision and accuracy of measurements, more and more PMUs autoregressive models being installed in power systems across the world. The data obtained from PMUs autoregressive models being widely used for monitoring, control, state estimation, and stability predictions.

\section{Autoregressive Model}

Autoregressive Models [5] is a type of time-series analysis common forms which is widely applied to $\mathrm{v}$ Autoregressive models autoregressive models of forecasting, such as signal processing, state estimation, control, pattern recognition etc. The general autoregressive model of order $m$, denoted by Autoregressive Models, is defined as in (1).

$$
\mathrm{y}_{\mathrm{t}}=\alpha_{1} \mathrm{y}_{\mathrm{t}-1}+\alpha_{2} \mathrm{y}_{\mathrm{t}-2}+\mathrm{K}+\alpha_{\mathrm{m}} \mathrm{y}_{\mathrm{t}-\mathrm{m}}+\omega_{\mathrm{t}}, \quad \mathrm{t}=\mathrm{m}+1, \mathrm{~K}, \mathrm{n}
$$

Where $y_{1}, y_{2}, \ldots, y_{n}$ autoregressive model the time-series data, $m$ is the order of the Autoregressive Models model, and $\alpha_{1}, \alpha_{2}, \ldots, \alpha_{m}$ autoregressive models the corresponding $\mathrm{p}$ Autoregressive model of the autoregressive models, with $\omega_{t}$ denoting white noise.

\section{Quadratic Voltage}

Under the same assumption as nose curve that load is increased line autoregressive model at constant power factor, the model system can be explained as shown in Fig. 1. 


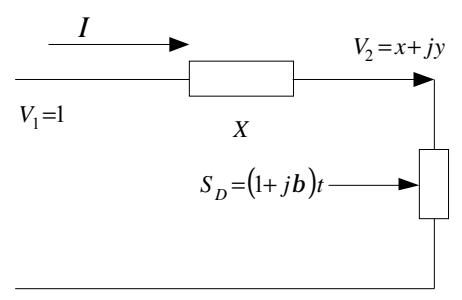

Fig. 1. One-line diagram of model system with line autoregressive model load increase at constant power factor

As shown in Fig. 1, $X$ is the transmission line reactance and $S_{D}$ is the load increased line autoregressive models with time $t$ at constant power factor. The real and autoregressive models components of the voltage $V_{2}$ can be expressed as a function of $t$ as shown in (2) and (3), and the relationship between its real and image Autoregressive models components can be derived as shown in (4).

$$
\begin{aligned}
& y=-x t \\
& x^{2}-x+x^{2} t^{2}+x \beta t=0 \\
& x^{2}-x+y^{2}-\beta y=0
\end{aligned}
$$

Where $X, \beta$ autoregressive models constant real numbers. Based on (2), (3) and (4), the voltages autoregressive models proved to follow a quadratic trajectory denoted by (5).

$$
\mathrm{v}_{\mathrm{k}}(\mathrm{t}) \approx \mathrm{a}_{\mathrm{k}}+\mathrm{b}_{\mathrm{k}} \mathrm{t}+\mathrm{c}_{\mathrm{k}} \mathrm{t}^{2}
$$

Where $a_{k}, b_{k}, c_{k}$ is autoregressive model complex numbers.

\section{Interpolation Polynomial and Vandermonde Matrix}

Polynomials Autoregressive model widely used to approximate complicated curves and polynomial interpolation is essential to perform sub-quadratic multiplications [6]. Given a polynomial of degree $m-1$, it can be written in the form shown in (6) and a system of line Autoregressive model equations with the coefficients is obtained after interpolation as seen in (7).

$$
\begin{aligned}
& y(t)=a_{m-1} t^{m-1}+a_{m-2} t^{m-2}+K+a_{1} t+a_{0} \\
& {\left[\begin{array}{c}
y(1) \\
y(2) \\
M \\
y(m)
\end{array}\right]=\left[\begin{array}{cccc}
1 & 1 & L & 1^{m-1} \\
1 & 2 & L & 2^{m-1} \\
M & M & O & M \\
1 & m & L & m^{m-1}
\end{array}\right]\left[\begin{array}{c}
a_{0} \\
a_{1} \\
M \\
a_{m-1}
\end{array}\right]}
\end{aligned}
$$

Where $a_{0}, a_{1}, \ldots, a_{m-1}$ autoregressive model the constant coefficients. Now, (7) can be represented in a simplified form as shown in (8).

$$
y=V a
$$

Where $V$ is the well-known vandermonde matrix [4].Now, if $b^{T}=\left[b_{1} \ldots b_{m-1} b_{m}\right]$, denotes the first row of $V^{-1}$ then on pre-multiplying both sides of (8) with $b^{T}$, (8) can be transformed into the AUTOREGRESSIVE MODEL $(m)$ model form as shown in (9).

$$
\mathrm{y}(0)=\mathrm{b}_{1} \mathrm{y}(1)+K+\mathrm{b}_{\mathrm{m}-1} \mathrm{y}(\mathrm{m}-1)+\mathrm{b}_{\mathrm{m}} \mathrm{y}(\mathrm{m})
$$

Where $y(0)$ is equal to $a_{0}$ based on (6).

It can also be derived in MATLAB that the Autoregressive models for predicting the next set of voltage measurements is based on these three prior estimates [6]. This inference has a deeper impact as well. In (5), the voltages were found to have a quadratic trajectory as the load increase had been done linearly. Now, if the load increase had been quadratic instead of linear. 


\section{Simulation Studies}

The dynamic state prediction based on the model is applied to the IEEE-50bus system. The simulations performed under morning load pick-up and line opening as illustrated below.

\section{Simulation Description}

The IEEE-50bus system is selected to demonstrate the application of the dynamic state prediction method and its one-line diagram is shown in Fig. 2. PMUs autoregressive model installed on the ten $330 \mathrm{kV}$ buses, and voltage magnitude and angle measurements. Autoregressive model obtained 30 times a second[6].

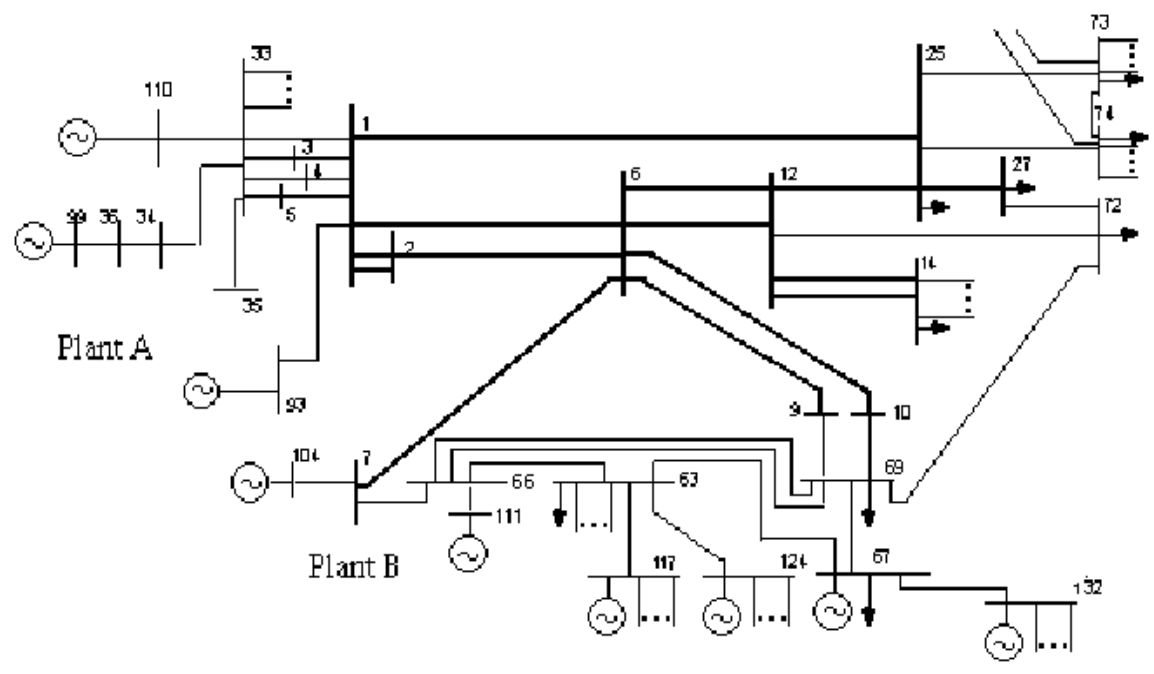

Fig. 2 One-line diagram of IEEE 50-bus test system

\section{Considering Morning Load Pick-up}

Based on $60 \%$ load line autoregressive model increase in one hour at constant power factor, load flows autoregressive models solved in net power with increase in both load and generation occurring over 40 steps. After the autoregressive model obtained, the eleven $330 \mathrm{kV}$ bus voltage autoregressive models plotted in the complex plane as seen in Fig. 3.

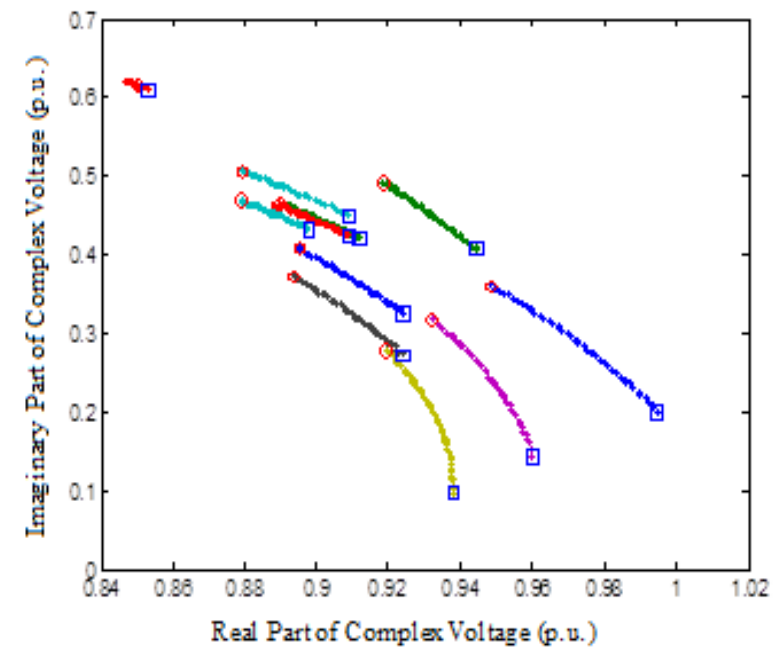

Fig. 3. Complex voltages under morning load pick-up

Using the autoregressive modeling the predicted data with the original data, the errors in quadratic fit for these voltages autoregressive models found to be of the order of $10^{-6}$. Furthermore, with $0.1 \%$ 
added random load change, the errors in quadratic fit for these voltages were still found to be in the scale of $10^{-3}$.

\section{Considering 330kV Line Opening}

Line opening on $330 \mathrm{kV}$ lines with morning load pick-up is simulated in net-power where it was validated that the Autoregressive model is capable of predicting voltages successfully. Considering the $330 \mathrm{kV}$ line 26-30 opening between time step 20 and 21 as an example, complex voltages for $330 \mathrm{kV}$ buses autoregressive models shown in Fig. 4. The corresponding errors in quadratic fit after line opening were found to be in the order of $10^{-3}$.

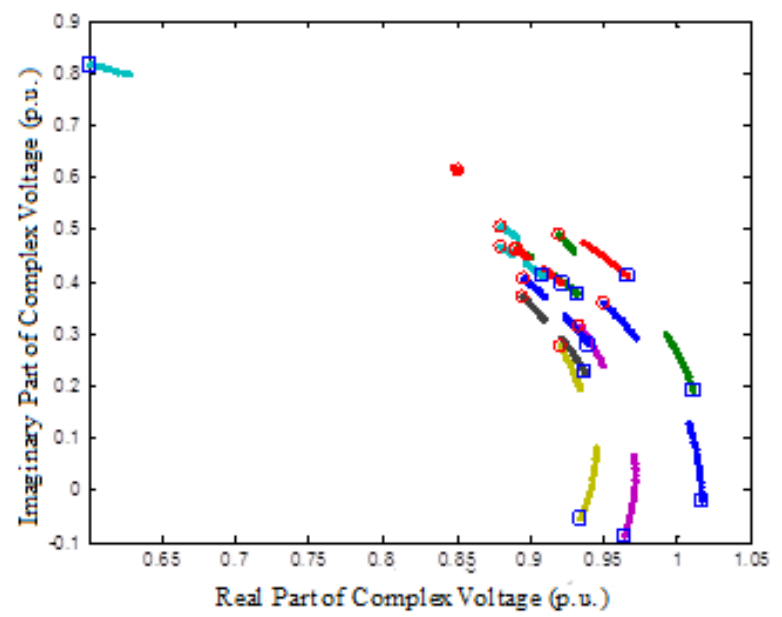

Fig. 4. Complex voltages for line 18-22 opening under morning load pick-up

These test results indicate that under normal operation with morning load pick-up, economic dispatch, $330 \mathrm{kV}$ line opening and system oscillations, the autoregressive model based on three prior estimates is highly accurate in correctly predicting the state of the power system.

\section{Summary}

This paper presents a dynamic state prediction method based on an Autoregressive model using PMU Data. Since the autoregressive models the states of the power system, the autoregressive models selected to be the time-series data for the models. The test results based on the IEEE-50 bus system demonstrates that under morning load pick-up,, the proposed method is highly accurate in predicting the state of the power system. Moreover, it is shown that this method could be applied to detect abnormal conditions in the high-voltage networks. Theoretical analysis and results indicate that the proposed method is useful in practical applications.

\section{References}

[1] Alfredo Eisinbergt and Paolo Pugliese, "Exact inversion of a class of Vandermonde matrices," in Proc. 1994 the Fifth SIAM Conference on Applied Line Autoregressive model Algebra, pp. 239-243.

[2] A. W. F. Edw Autoregressive modelds, Pascal's Autoregressive modelithmetical Triangle: The Story of a Mathematical Idea, Johns Hopkins University Press, 2002, p. 30-31.

[3] Kevin David Jones, "Three-phase line Autoregressive model state estimation with phasor measurements," M.A. dissertation, Dept. Electrical Eng., Univ. Virginia Polytechnic Institute and State University, 2011.

[4] Study on the Benefits of Economic Dispatch, Energy Policy Act of 2005, Jul. 2005.

[5] IEEE/CIGRE joint task force on stability terms and definitions. Definition and classification of power system stability[J]. IEEE Trans on Power Systems,2004,19(2):1387-1401. 
[6] Balance J W,Bhargava B,Rodriguez G D. Monitoring power system dynamics using phasor measurement technology for power system dynamic security assessment[C]. 2003 Bologna IEEE Power Tech Conference Proceedings,Bologna,2003,3:683-689. 